\title{
A Computer Vision System for Color Grading Wood Boards Using Fuzzy Logic
}

\author{
João Faria*, Teresa Martins**, Manuel Ferreira*, Cristina Santos* \\ University of Minho - Industrial Electronics Department - Guimarães, Portugal. \\ *Department of Industrial Electronics \\ University of Minho \\ Campus de Azurem - Guimarães, Portugal \\ Telf: +351253510190; fax: +351253510189; email: jbravofaria@gmail.com, \\ mjf@,dei.uminho.pt, cristina@,dei.uminho.pt \\ **Enermeter, Sistemas de Medição, SA \\ Praceta das Agras, 26 \\ Celeiros - Braga, Portugal \\ Telf: +351253287237; fax:+351253287238; email: tmartins@enermeter.pt
}

\begin{abstract}
This paper describes the development of a project based on Fuzzy Logic applied to color histograms for color grading wood boards. During this development a fuzzy min-max inference system, with bell membership functions adjusted to the histogram, was implemented and evaluated. This system performs image matching. Images given as training samples are used to define the classes that determine the several wood board color intervals. In that way the system is capable to classify an image by its color in order to group wood boards with the same tonalitycolor in a short period of time.
\end{abstract}

\section{INTRODUCTION}

New developments in computer vision raise the possibility to create automatic systems capable of performing the same tasks as human workers, more quickly and with more accurate results, increasing productivity and assuring a more correct evaluation of products quality. Consequently an increased interest has been expressed by industrials of different sectors end users and equipment manufactures - in this technology. This is also the case of the wood transformation sector that shows plainly awareness of computer vision techniques to perform quality control of its products.

This paper describes a system based on fuzzy logic applied to color histograms for color grading wood boards. It is able to classify the several board images according to their color in a short period of time, in order to group wood boards with the same tonality-color and therefore it fulfills industrial requirements. Figure 1 shows distinctive images of wood boards with typical color and texture used in the furniture industrial sector. The wood boards have, in general, dimensions of $300 \times 2000 \mathrm{~mm}$.

While performing automatic visual inspection using a computer vision system, constrains related with the system's layout must be considered. To avoid reflections or shadows and therefore to assure a good image for analysis, it is imperative to evaluate the type of cameras and the type of illumination as well as their pose. The industrial setup for this system should consist on a linear CCD camera placed above a conveyor belt and a high frequency fluorescent light (Figure 2). To simulate it, in order to validate the algorithms and the overall approach on laboratory, the board images were acquired using a general purpose scanner.

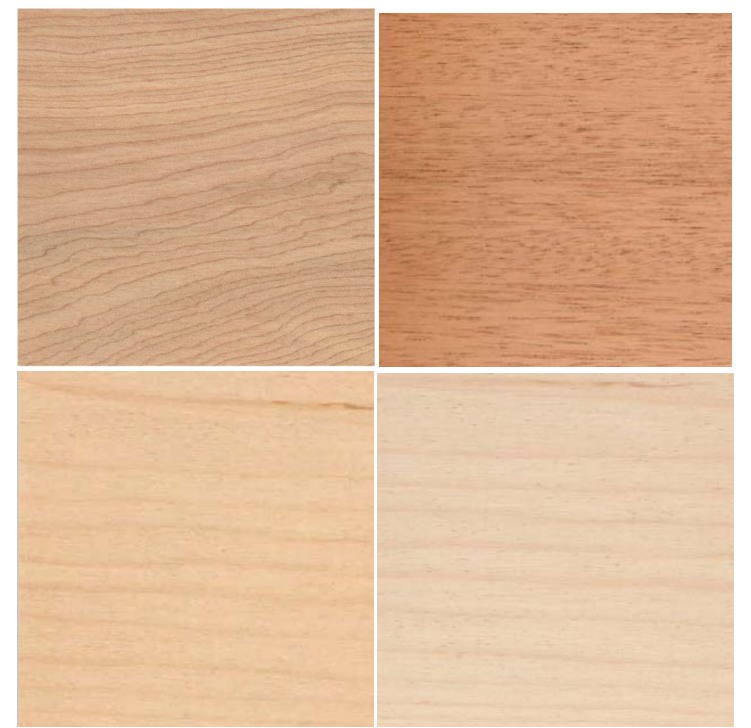

Fig. 1. Images of typical samples of wood boards.

Nowadays different methods can be found in the literature for color grading and surface inspection. In the purpose of this subject it is important to mention Self-Organizing Maps (SOM), also known as Kohonen's Maps [1,2,3,4] and Fuzzy Logic $[5,6,7]$. Very good results can be achieved using SOM 
for color grading and surface inspection [8], therefore it is more used in applications that require accurate results with high-dimensional data. Fuzzy logic applied to the histograms of an image presents also good results in color matching and it has a quite simpler implementation, which is an important factor when dealing with industrial applications. This attribute was the main reason to base the present solution on fuzzy logic.

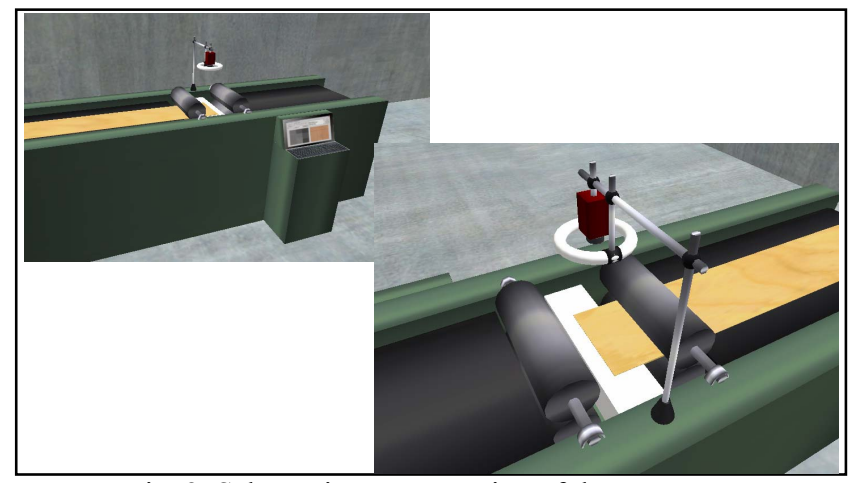

Fig. 2. Schematic representation of the system setup.

\section{METHODS}

The fuzzy inference system used in this application was the min-max inference system $[9,10]$. To apply the min-max fuzzy inference system the universe of discourse must be divided in several classes. Each class is defined through the behavior of a set of features (variables). For each feature, of each class, a fuzzy function - fuzzy membership function - must be specified in order to characterize that variable. Figure 3 shows an application example of the min-max inference system.

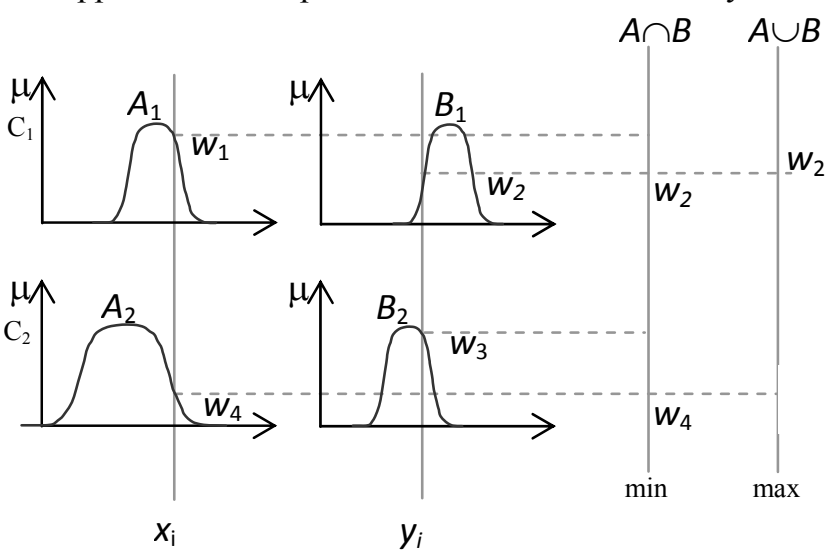

Fig. 3. Min-Max fuzzy inference system.

In this example two variables, $x$ and $y$, are modeled into two classes $C_{1}$ and $C_{2}$ by the membership functions $\mathrm{A}_{1}, \mathrm{~B}_{1}$ and $\mathrm{A}_{2}$, $\mathrm{B}_{2}$, respectively. When a pair of values $\left(x_{i}, y_{i}\right)$ is applied to the inference system, the first operation is to determine the respective membership grade for each membership function. Then, for each class $\left(C_{1}\right.$ and $\left.C_{2}\right)$ it is determined the minimum value $w_{2}$ and $w_{4}$, respectively. The pair will belong to the class with the higher minimum value, in this case $\mathrm{w}_{2}$.
In the color grading system the classes correspond to the different types of wood boards and the feature extracted from that classes are the different color channels histogram.

The first step was to find the color spaces able to differentiate the several wood images based on its histograms properties. The color spaces selected were HSV and CIE $\mathrm{L} * \mathrm{a}^{*} \mathrm{~b}$ for the reason that, considering the histograms it was possible to extract enough features that gave the system the capacity to distinguish images that looked similar to human eye $[11,12]$. Also, these color spaces are more uniform than RGB and are less sensitive to illumination drifts.

Color space HSV is a device dependent model $[12,13]$ that makes use of three concepts: Hue; Saturation and Value, and is a nonlinear transformation of RGB color space (figure 4). Hue corresponds to the dominate wavelength of the scene spectrum and ranges from $0^{\circ}$ to $360^{\circ}$. Saturation is related with the purity of the color, meaning the quantity of white. It ranges from 0 (no color) to 100 (pure color). Value is related with the brightness of the color, meaning the intensity of the electromagnetic energy, and it ranges from 0 (black) to 100 (white).

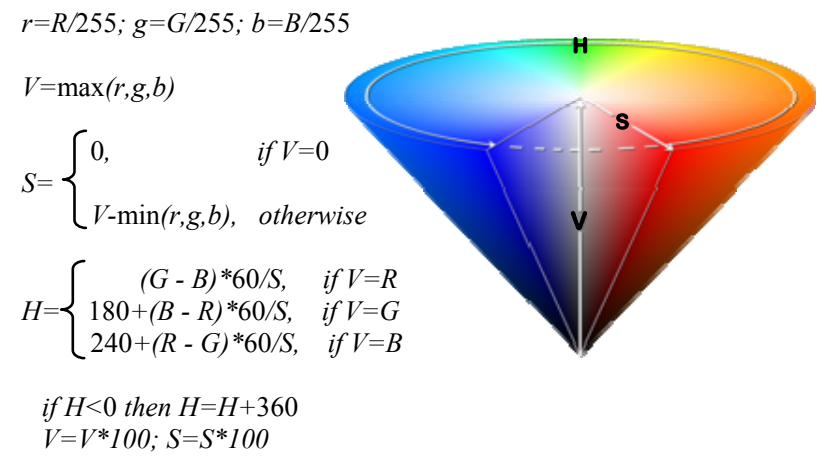

Fig. 4. Conical representation of the HSV model and RGB to HSV conversion equations.

Color space CIE L*a*b* describes all the colors perceived by the human eye and is a device independent color model $[14,15,16,17]$. The coordinates represent the lightness of the color $-\mathrm{L}^{*}$ (from black $\mathrm{L}^{*}=0$ to white $\mathrm{L}^{*}=100$ ), and its coloropponent dimensions (figure 5).

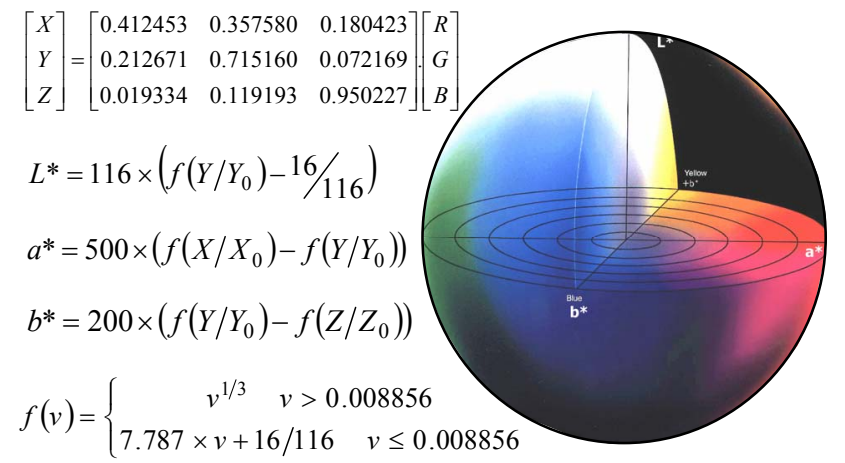

Fig. 5. Sphere representation of the CIE L*a*b* model and conversion equations. $X_{0}, Y_{0}, Z_{0}$ is the device dependent white point. The conversion from RGB to XYZ color space makes use of CIE Rec. 709. 
$\mathrm{a}^{*}$ is the Red/Green opponent channel; negative values indicate green, while positive values indicate red. $b^{*}$ is the Yellow/Blue opponent channel; negative values indicate blue, while positive values indicate yellow.

The min-max membership functions must model the color histograms of the images. Six variables will be defined, one for each color channel: H, S, V, L*, a* and $b^{*}$. The number of classes is dependent on the number of wood board types to classify.

As an example, figure 6 shows the histogram for each color channel obtained from two wood types.
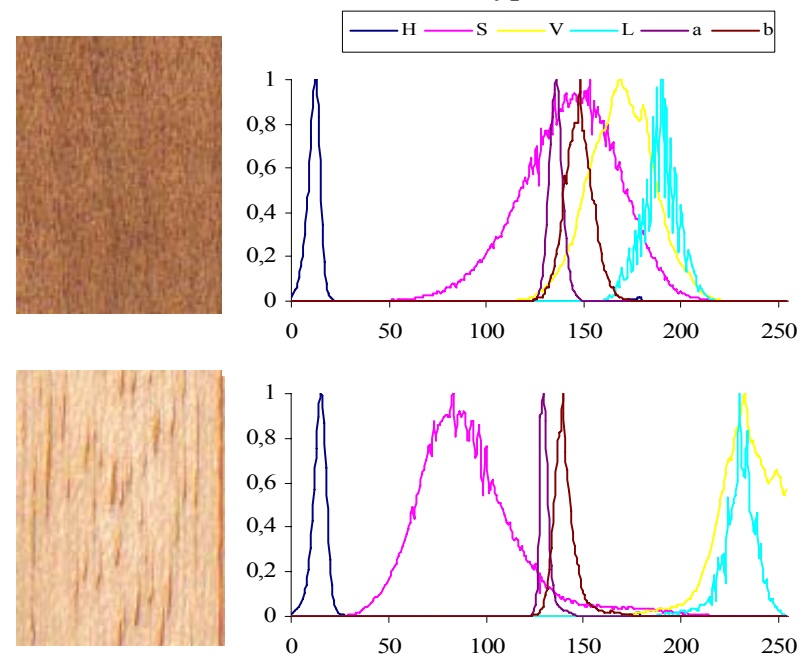

Fig. 6. Two wood board images and the respective histograms of HSV and CIEL*a*b* color spaces.

After creating the histograms it is necessary to determine the fuzzy membership function that represents them best training phase. By analyzing the histograms of different wood board images the solution to achieve a more accurate representation was to approximate each histogram to bell functions. The approximation was made by adjusting the parameters of the bell function (figure 7).

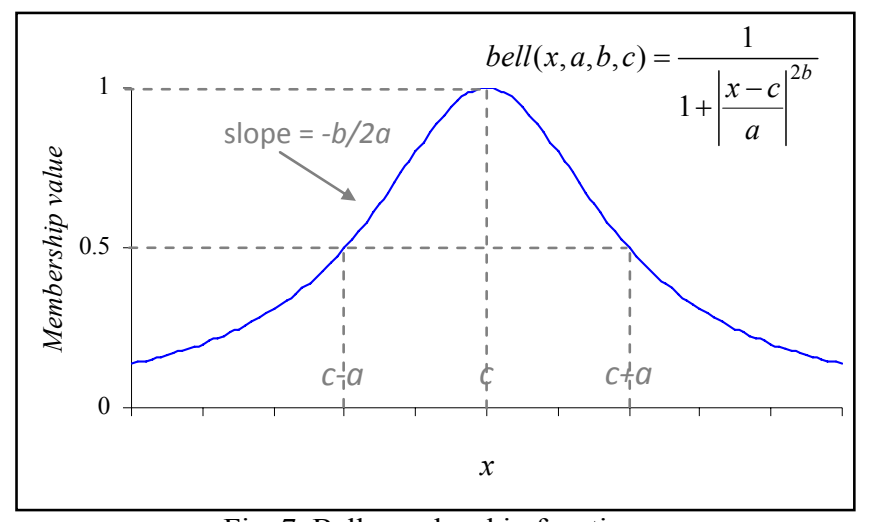

Fig. 7. Bell membership function.

While approximating the functions it was taken in account that the bell function should embrace all the real histogram function. Some examples of different histograms and the approximated functions can be observed in figure 8 .
The greatest difficulty to overcome in the present approach is the training method, since it must be reliable in an industrial context. This implies that the parameters of the bell functions should be automatically determined. Besides, this kind of systems has to deal with a significant number of classes that should be modeled in short periods of time, and for that a simple training procedure must be used. In a first approach the maximum, the difference between crossover points and the asymmetry values, extracted from each histogram, were used to adjust the bell function parameters $c, a$ and $b$ respectively. However, in order to diminish the board texture effect a smooth and a morphological close filters were applied to the image which allowed the fixing of the $b$ parameter to value 1 . Figure 9 shows the application of these filters to the samples represented in figure 6 from which result more symmetric histograms.
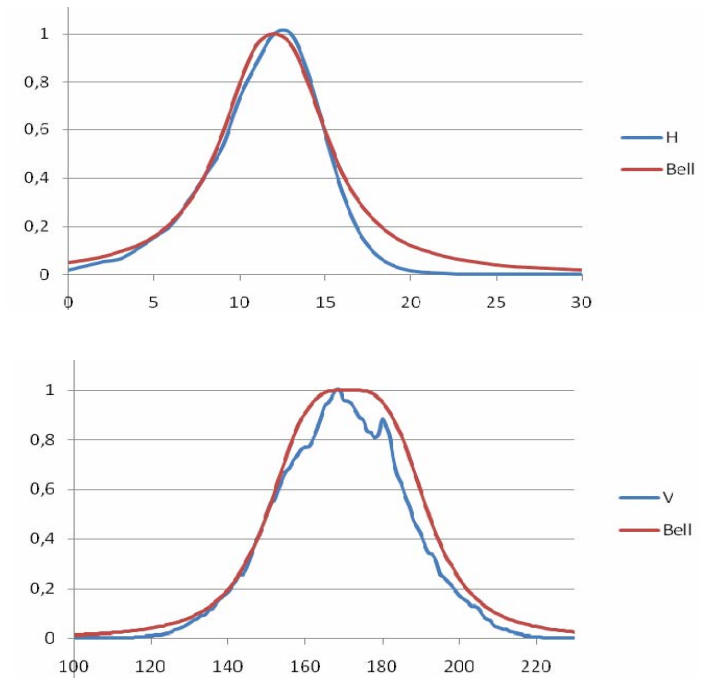

Fig. 8 - Example of a bell approximation to the $\mathrm{H}$ and $\mathrm{V}$ histograms.
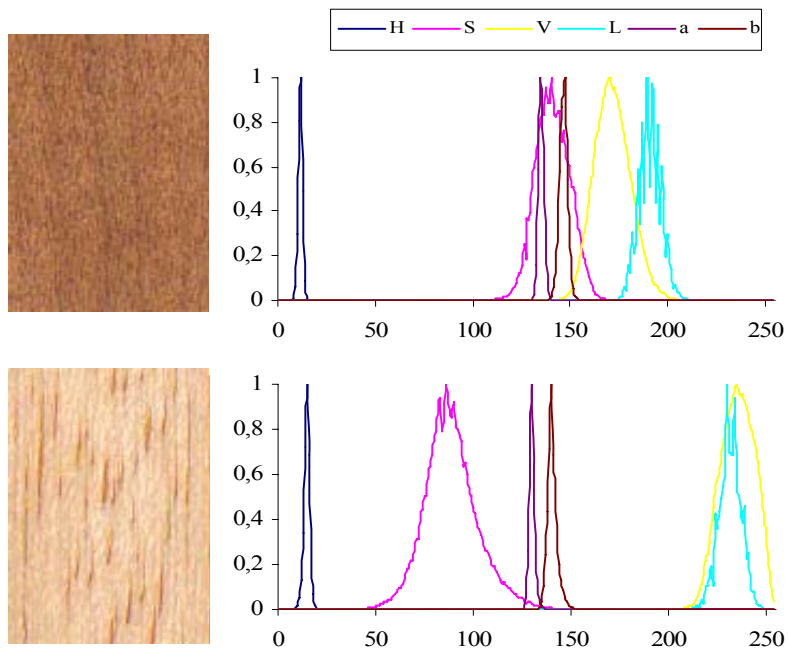

Fig. 9. Two filtered wood board images and the respective histograms of HSV and CIEL*a*b* color spaces. 


\section{RESULTS AND DISCUSSION}

During the training phase - the Bell approximation to the histograms - an image of a piece of wood board, with size $100 \times 150 \mathrm{~mm}$, with resolution of 6 pixels $/ \mathrm{mm}$, was used to extract the histogram of each channel.

In the testing phase, the board image was divided in a number of overlapping windows with dimension D (Fig. 10). Since the classification efficiency of the system depends on the $D$ parameter, different values were experimented $(D=100$; $\mathrm{D}=200 ; \mathrm{D}=400) . d x$ and $d y$ are the displacements relatively to the previous window; their values affect the segmentation accuracy. A value of $\mathrm{D} / 2$ was used for both parameters.

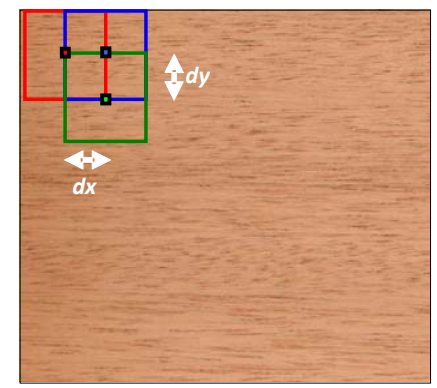

Fig. 10. Decomposition process of the image during the testing phase: Red window $d x=0, d y=0$; Blue window $d x=\mathrm{D} / 2, d y=0$; Green window $d x=\mathrm{D} / 2, d y=\mathrm{D} / 2$.

The system was evaluated for the following three major types of wood: cherry tree, beech tree and oak, that will be designated herein by $c t, b t$ and ot respectively. For each type, distinct color classes were defined in such a way to observe the industrial needs. Specifically five classes for $o t$, two classes for $b t$ and three classes for $c t$ were established. For each color class three samples were used, coded as followed: $n T s ; T-$ type of tree $\{c t, b t, o\} ; n$ - color class $\{1 . .5\} ; s$ - sample $\{1 . .3\}$.

Each sample was considered by the inference systems as a class. The purpose of this was to evaluate how the system behaves in extreme conditions, meaning closed color classes. So, it results in 30 wood classes presented to the inference system.

Figure 11 shows the application of the fuzzy min-max to a sample image (1ct2) decomposed in windows of $\mathrm{D}=100$ pixels.

Figure $11 \mathrm{a}$ is part of the $1 c t 2$ image in which the decomposition windows are marked. Figure $11 \mathrm{~b}$ presents the segmentation result. The numbers indicate that the fuzzy minmax procedure segments the image in different classes: number 3 refers to class $1 c t 2$, number 4 to class $1 c t 3$ and number 16 to class 4 ot2. Figure $11 \mathrm{c}$ and $11 \mathrm{~d}$ show part of the images of the classes 1 ct 3 and 4ot2, respectively.

Apparently this segmentation led to a bad classification of the $1 c t 2$ sample. However $1 c t 3$ belongs to the same type of wood and to the same color class, which means that from the industrial point of view the segmentation was correctly performed. Therefore the error in the segmentation occurs only in the window with the number 16, which is classified as belonging to the sample 4 ot 2 .
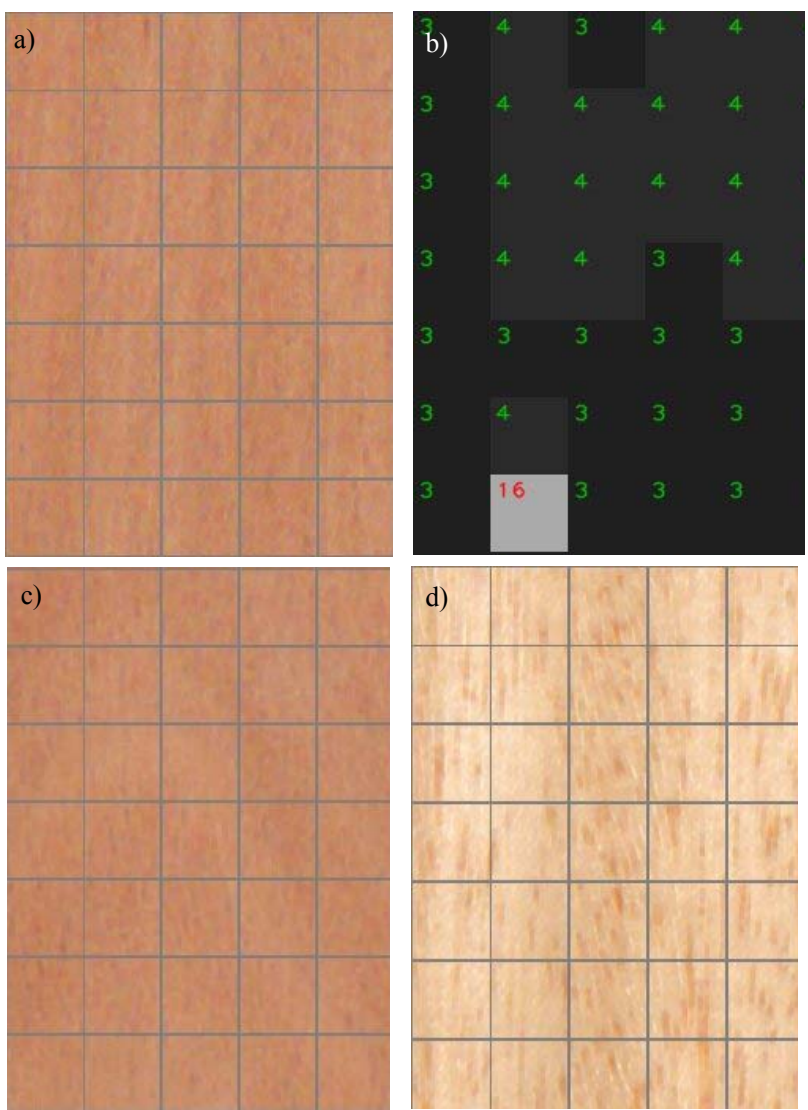

Fig. 11. Result of the application of the system to the $1 c t 2$ sample image showing the windows decomposition with $\mathrm{D}=100$ and the segmentation result.

Figure 12 shows the system rates for the universe of sample images that were decomposed in windows of 100x100 pixels.

Depending on the type of wood boards the classification rate varies from $30 \%$ to $84 \%$. These low rates can be comprehended by analysing the chart of figure 13, which presents the results for oak samples.

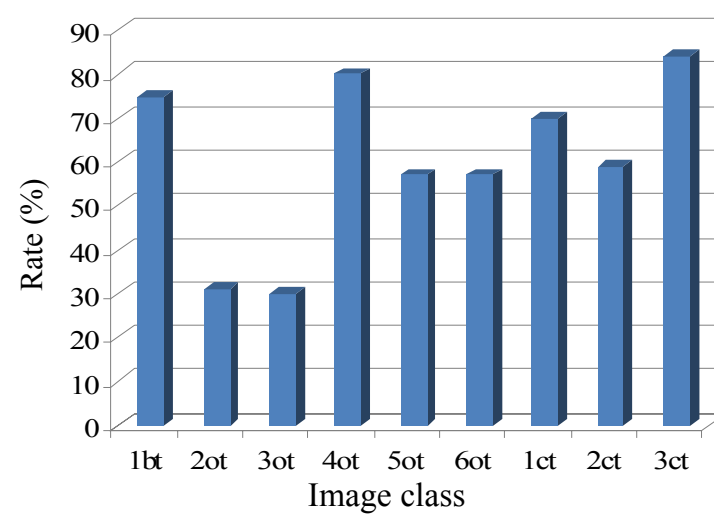

Fig. 12. Classification rates obtained with $\mathrm{D}=100$.

This chart represents the correct sample classifications, the correct color classifications (sample classified as belonging to the correct type of wood and to the correct color class), the correct wood classifications (sample classified as belonging to 
the same type of wood) and the bad classifications (sample classified as other wood types). From the industrial point of view correct color classifications are good classifications.

Bad classification rates are low for the generally of the samples. For some samples the system classifies correctly the wood type but gives poor color and sample classifications (for example 2ot2).

These poor classification rates are due to the texture present in the images, and consequently are influenced by the size of the decomposition window. In order to diminish this effect, the size of the window (D parameter) has been enlarged.

Table I and figure 14 show the classification rates for windows of size 100, 200 and 400 pixels. Raising the D value leads to an increase in the classification rates to values higher than $95 \%$. Depending on the type of wood boards, and consequently on its natural texture, acceptable rates can be achieved with lower windows size.

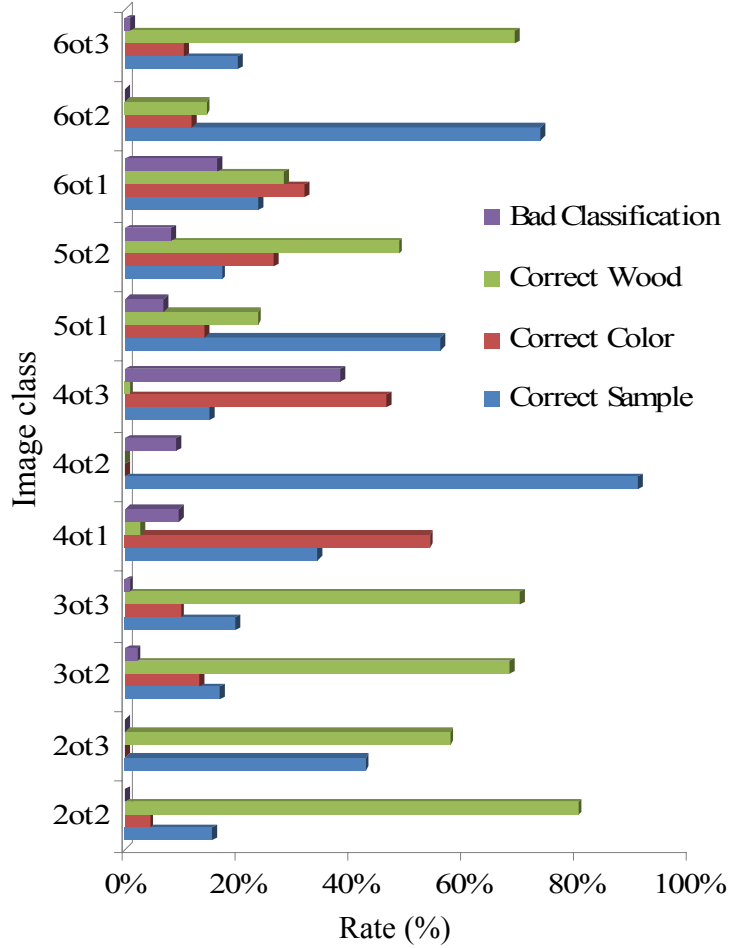

Fig. 13. Classification rates obtained for oak samples with $\mathrm{D}=100$.

\section{CONCLUSION}

The present solution classifies wood boards based on its color histograms, specifically $\mathrm{H}, \mathrm{S}, \mathrm{V}, \mathrm{L}^{*}, \mathrm{a}^{*}$ and $\mathrm{b}^{*}$ channels. The classifier is a fuzzy min-max inference system that uses bell membership functions adjusted to each histogram. The pre-processing procedure (a smooth plus a close filter) diminishes the board texture which simplifies the traning phase and also increases the robustness of the solution.

The evaluation of the system is based on its accuracy and training method. Using the Bell approximation the obtained results were very satisfactory once the system was capable to distinguish all the image classes that were used to train and test it. While evaluating the test images, the best results were obtained with a window decomposition of 400x400 pixels, since it makes the system even less sensitive to the natural wood texture. In this condition the classifier reveals high classification rates, higher than $95 \%$.

TABLE I

Classification rates for different windows size $(D)$

\begin{tabular}{c|ccc} 
Class & 100 & 200 & 400 \\
\hline $1 b t$ & 75 & 94 & 100 \\
$2 o t$ & 31 & 56 & 95 \\
$3 o t$ & 30 & 67 & 96 \\
$4 o t$ & 80 & 94 & 98 \\
$5 o t$ & 57 & 82 & 96 \\
$6 o t$ & 57 & 100 & 100 \\
$1 c t$ & 70 & 86 & 96 \\
$2 c t$ & 59 & 80 & 95 \\
$3 c t$ & 84 & 86 & 98
\end{tabular}

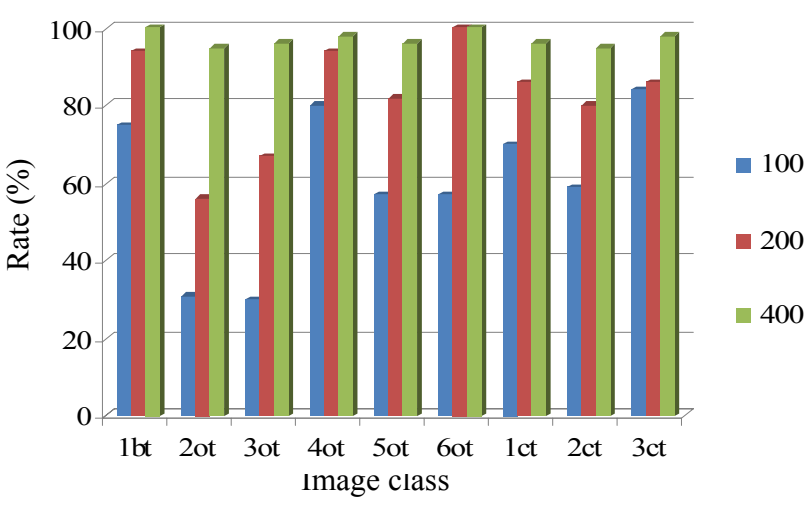

Fig. 14. Classification rates for different windows size $(D)$

\section{REFERENCES}

[1] Konstantinos Zagoris, Nikos Papamarkos and Ioannis Koustoudis, "Color Reduction Using the Combination of the Kohonen SelfOrganized Feature Map and the Gustafson-Kessel Fuzzy Algorithm", Machine Learning and Data Mining in Pattern Recognition, Springer, Vol. 4571, pp. 703-715, 2007, ISBN 9783-540-73498-7.

[2] Lescure, P., Meas Yedid, V., Dupoisot, H., and Stamon, G. "Color segmentation of biological microscopic images," in Proceedings of the SPIE - The International Society for Optical Engineering, Vol. 3647, pp. 182-93, 1999.

[3] Likas, A. "A reinforcement learning approach to online clustering". Neural Computation, 11:1915-32, 1999

[4] Max Dennis Luesebrink, "Self-Organising Maps For Colour Recognition", available in URL: http://aidepot.com/Tutorial/SomColour.html; (27/12/2006).

[5] A Doncescu, J Aguilar-Martin, JC Atine, "Image color segmentation using the fuzzy tree algorithm T-LAMDA", Fuzzy Sets and Systems Vol. 158, Issue 3, pp. 230-238, February 2007.

[6] Cheng H.D.1; Li J, "Fuzzy homogeneity and scale-space approach to color image segmentation" Pattern Recognition, Elsevier, Vol. 36, No. 7, pp. 1545-1562(18), July 2003. 
[7] Alexandre da Silva Simões, Anna Helena Reali Costa, Marco Túlio Carvalho de Andrade, "Utilizando Um Classificador Fuzzy Para A Seleção Visual De Laranjas", in Workshop de Computação WORKCOMP'2001, Instituto Tecnológico de Aeronáutica - ITA, São José dos Campos, SP. 2001.

[8] Matti Niskanen, "A Visual Training Based Approach To Surface Inspection", Acta Universitatis Ouluensis C 186, 125 p, 2003, ISBN 951-42-7066-5.

[9] G.A. Ruz and P.A. Estévez, "Image segmentation using fuzzy min-max neural networks for wood defect detection". Intelligent Production Machines and Systems-First I*PROMS Virtual Conference, D.T Pham, E.E. Eldukhri and A.J. Soroka (eds.), pp. 183-188, July 2005. Elsevier Ltd.

[10] J. R. Jang, C. T. Sun, E. Mizutani, Neuro-fuzzy and soft computing, a computational approach to learning and machine intelligence, Prentice-Hall, 1997.

[11] Benjamin D. Zarit, Boaz J. Super, Francis K. H. Quek, "Comparison of Five Color Models in Skin Pixel Classification", in Proceedings of the International Workshop on Recognition, Analysis, and Tracking of Faces and Gestures in Real-Time Systems, p.58, September 1999.

[12] Raphael Gonzalez, Richard E. Woods, Digital Image Processing, 2nd ed. Prentice Hall Press, p. 295. 2002, ISBN 0201-18075-8.

[13] Donald Hearn, M. Pauline Baker, Computer Graphics Prentice Hall International, pp. 302-205, 1986, ISBN 0-13-165598-1.

[14] Dan Margulis. Photoshop Lab Color: The Canyon Conundrum and Other Adventures in the Most Powerful Colorspace, ISBN 0321356780 .

[15] Fred W. Billmeyer, Max Saltzman, Principles of color technology, 2ed: John Wiley \& Sons, 1981.

[16] Alexander Poularikas, Digital Color Imaging Handbook, CRC Press, 2003.

[17] Jon Y. Hardeberg, "Acquisition and reproduction of color images: Colorimetric and Multispectral Approaches", $\mathrm{PhD}$ Thesis, USA, 2001, Available in URL:http://www.Dissertation.com, $(10 / 06 / 2003)$ 